\title{
Efecto del estadio de desarrollo de la onda folicular sobre la respuesta ovárica y tasa de recuperación y calidad de embriones en alpacas
}

\author{
Effect of the development stage of the follicular wave on ovarian response, and \\ rate of recovery and quality of embryos in alpacas \\ Josías Ascencio S. ${ }^{1}$, Wilfredo Huanca L., ${ }^{13}$, Jesús Turín V. ${ }^{1}$, Camilo Mamani M. ${ }^{1}$, \\ Aída Cordero R. ${ }^{2}$, Fred Hilari O. ${ }^{1}$
}

\section{Resumen}

Se evaluó el efecto del estadio de desarrollo de la onda folicular (fase de crecimiento, estático y regresión del folículo dominante) previo a la monta natural sobre la tasa de ovulación, y tasa de recuperación y calidad embrionaria en alpacas. Se trabajó con 51 alpacas hembra con presencia de folículo dominante mayor o igual a $7 \mathrm{~mm}$, las que fueron sincronizadas con un análogo de GnRH. Los grupos fueron $T_{1}(n=16)$ : Fase crecimiento; $\mathrm{T}_{2}(\mathrm{n}=19)$ : Fase estática y $\mathrm{T}_{3}(\mathrm{n}=18)$ : Fase regresión. El día de la monta fue considerado como día 0 . Se hicieron evaluaciones ecográficas en el día 2 (ocurrencia de la ovulación) y en el día 7 (para medir el tamaño del cuerpo lúteo). El lavado uterino se realizó a los 7 días del servicio. Dos animales de $T_{1}$ no llegaron a ovular. No hubo diferencias significativas entre grupos por tamaño del folículo dominante en el momento del servicio, tasa de ovulación ni por tamaño del cuerpo lúteo en el día del lavado uterino. En el 52.9\% ( $n=27)$ de las alpacas servidas se recuperaron 27 embriones sin diferencias significativas entre tratamientos; no obstante, los embriones de $\mathrm{T}_{1} \mathrm{y} \mathrm{T}_{2}$ fueron de mejor calidad que los de $\mathrm{T}_{3}$ $(\mathrm{p}<0.05)$.

Palabras clave: alpacas; estadio folicular; calidad embriones; cuerpo lúteo

\footnotetext{
${ }^{1}$ Laboratorio de Reproducción Animal, Facultad de Medicina Veterinaria, Universidad Nacional Mayor de San Marcos, Lima, Perú

${ }^{2}$ Departamento de Nutrición, Facultad de Zootecnia, Universidad Nacional Agraria La Molina, Lima, Perú

${ }^{3}$ E-mail: whuancal@unmsm.edu.pe
} 
The effect of the stage of development of the follicular wave (growth phase, static and regression of the dominant follicle) prior to the natural mounting on the ovulation rate, and the recovery rate and embryo quality in alpacas was evaluated. It was used 51 female alpacas with presence of dominant follicle greater than or equal to $7 \mathrm{~mm}$, which were synchronized with a GnRH analogue. The groups were T1 $(n=16)$ : growth phase; T2 ( $n=19)$ : Static phase and T3 ( $=18)$ : Regression phase. The day of the copula was considered as day 0 . Ultrasonographic evaluations were conducted on day 2 (occurrence of ovulation) and on day 7 (to measure the size of the corpus luteum). The embryo recovering was done 7 days after the service. Two animals of $\mathrm{T}_{1}$ did not ovulate. There were no significant differences between groups by size of the dominant follicle at the time of service, ovulation rate or size of the corpus luteum on the day of embryo recover. In $52.9 \%(\mathrm{n}=27)$ of the alpacas, 27 embryos were recovered without significant differences between treatments; however, the embryos of $T_{1}$ and $T_{2}$ were of better quality than those of $\mathrm{T}_{3}(\mathrm{p}<0.05)$.

Key words: alpaca; follicular stage; embryo quality; corpus luteum

\section{INTRODUCCIÓN}

Una actividad socioeconómica importante para el poblador altoandino es la crianza de camélidos sudamericanos (CSA) (Fernández-Baca, 1971). El ecosistema donde habitan estos animales está por encima de los $4000 \mathrm{msnm}$, con pastos de baja calidad nutricional. Las alpacas presentan un pobre comportamiento reproductivo con una baja fertilidad, atribuida a una alta tasa de mortalidad embrionaria (Novoa, 1991). Aun así, esta especie ha sido capaz de adaptarse a este hábitat para producir fibra de gran calidad y carne altamente proteica (FernándezBaca, 1991).

Los CSA domésticos como la alpaca (Vicugna pacos) y la llama (Lama glama) y las especies silvestres como la vicuña (Vicugna vicugna) y el guanaco (Lama guanicoe) (Marín et al., 2007) son considerados especies de ovulación inducida, donde el factor principal que desencadena el fenómeno de ovulación está dado por la cópula (Fernández-Baca et al., 1972). El desarrollo de la onda folicular en alpacas se da de manera alterna en ambos ovarios, habiéndose detectado la presencia del folículo dominante en ambos ovarios en el $85 \%$ de los casos (Fernández-Baca, 1993) y el cuerpo lúteo posterior a la ovulación en el ovario derecho en el $51 \%$ de las observaciones, en el ovario izquierdo en el $47 \%$ y en ambos ovarios en el $2 \%$ de las llamas (Bravo et al., 1990; Sumar, 2000).

La tasa de mortalidad embrionaria puede llegar hasta el $50 \%$ durante los primeros 35 días de gestación en la alpaca, de allí que solo entre el 40 y $50 \%$ de las hembras en edad reproductiva pueden producir una cría al año (Fernández Baca et al., 1970). Una alternativa tecnológica para mejorar la eficiencia reproductiva en las alpacas es el uso de la transferencia de embriones (Ratto et al., 2013), aunque la tasa de recuperación de embriones es de 4.8 por animal (Huanca et $a l ., 2009)$ en llamas y 2.7 embriones/animal en alpacas (Huanca, 2008).

Los folículos en diferentes estadios contienen ovocitos que pueden variar en sus ca 
racterísticas y que pueden afectar la calidad de los embriones recuperados. El presente estudio tuvo como propósito evaluar el efecto del estadio de desarrollo de la onda folicular sobre la respuesta ovárica, tasa de recuperación y calidad de embriones en alpacas, buscando determinar posibles diferencias del estadio de desarrollo de la onda folicular sobre la calidad de ovocitos y el desarrollo embrionario temprano.

\section{MATERIALES y Métodos}

\section{Lugar de Estudio}

El estudio fue realizado entre los meses de enero y abril de 2015 en el Centro de Investigación y Producción Quimsachata, anexo de la Estación Experimental ILLPA, del Instituto Nacional de Innovación Agraria (INIA), en el distrito de Cabanillas, provincia de San Román, departamento de Puno, Perú.

\section{Evaluación Ecográfica Inicial}

La selección de las hembras se hizo con base a la presencia de folículos ováricos $\geq 7 \mathrm{~mm}$ de diámetro, utilizando un ecógrafo ALOKA SSD500 equipado con un transductor lineal de 7.5 Mhz. Se utilizaron 53 alpacas adultas de 5-8 años de la variedad Huacaya, vacías, con descendencia registrada y sin cría al pie. Se les administró $1 \mathrm{ml}$ de un análogo de GnRH (0.0042 $\mathrm{mg}$ de acetato de buserelina) (Conceptal, Intervet, Alemania), vía intramuscular, para la inducción de ovulación y sincronización de la onda folicular. Los animales fueron nuevamente evaluados por ecografía a las 48 horas, para verificar la ovulación, con base al criterio de la desaparición del folículo dominante previamente determinado.

\section{Diseño Experimental}

Los animales fueron sometidos a ecografías diarias, a partir del día 9 de la sincronización de la ovulación, para determi- nar la presencia de folículos $\downarrow 7 \mathrm{~mm}$ y distribuirlos a los tratamientos según el estadio de crecimiento, estático o de regresión del folículo dominante, debiendo permanecer en dichos estadios al menos durante dos días consecutivos. Con base a ello, los animales fueron distribuidos en $T_{1}$ : fase de crecimiento $(n=16) ; T_{2}$ : fase estática $(n=19) ; T_{3}$ : fase de regresión $(\mathrm{n}=18)$, correspondiendo el Día 0 al día del empadre.

El empadre controlado se hizo luego de la segunda ecografía, una vez que los grupos fueron determinados, por un tiempo mayor a 15 minutos para tratar de asegurar la inducción de la ovulación. Los machos empleados pertenecían al plantel de reproductores y tenían descendencia registrada. El día de la monta fue considerado el día 0 . Las evaluaciones ecográficas posteriores se realizaron al día 2 (ocurrencia de la ovulación) y el día 7 (medición del tamaño del cuerpo lúteo), haciendo además el lavado de los cuernos uterinos (Figura 1).

\section{Recuperación y Calidad de Embriones}

La recuperación de los embriones se realizó el día 7 posterior a la monta con base al protocolo descrito por Huanca et al. (2009). Previamente se hizo la evaluación ecográfica para determinar el tamaño del cuerpo lúteo. Para esto, los animales fueron sujetados en un brete y se les colocó anestesia epidural ( $2 \mathrm{ml}$ de lidocaína $2 \%$ ) entre la última vértebra sacra y la primera coccígea. Para la recuperación de embriones se procedió al lavado de los cuernos uterinos con una pipeta Foley N. ${ }^{\circ} 16$ e infusión de $100 \mathrm{ml}$ de solución de lavado (fosfato buffer salino + antibiótico gentamicina). La solución recuperada se colocó en el filtro EmCon (Agtech) para facilitar la separación del embrión del medio, la cual fue depositada en placas Petri para proceder a la búsqueda de los embriones con ayuda de un estereoscopio Nikon. Los embriones se clasificaron usando la escala mencionada en el manual de la International Embryo Transfer Society - IETS 


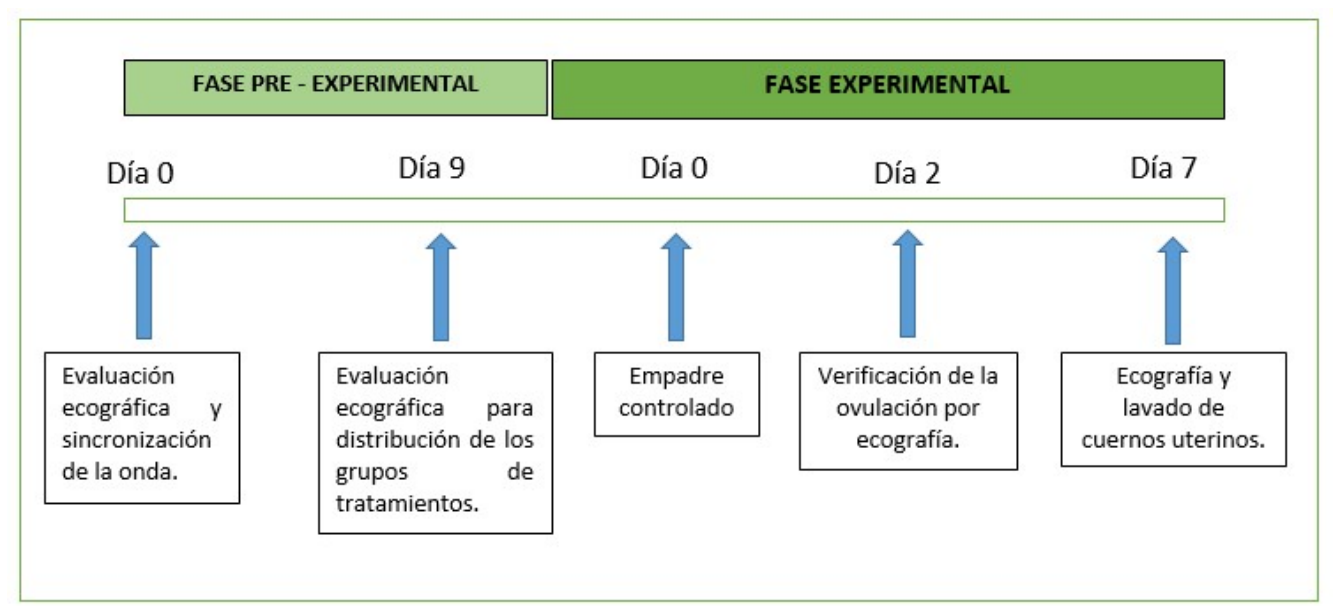

Figura 1. Procedimiento experimental

(Stringfellow y Givens, 2011), que clasifica los embriones en cuatro calidades:

- Excelente: Embrión ideal, esférico, simétrico, con células de tamaño, color y textura uniforme.

- Bueno: Embrión con pequeñas imperfecciones con algunas blastómeras de formas irregulares.

- Regular: Embrión con presencia de blastómeras de formas irregulares, con presencia de vesículas y algunas células degeneradas.

- Pobre: Embrión con numerosas blastómeras de formas irregulares, células degeneradas, células de diversos tamaños.

\section{Análisis Estadístico}

Los datos fueron analizados utilizando el paquete estadístico Stata 11.0 (Stata Corp). La prueba del análisis de varianza de una sola vía fue utilizada para determinar la diferencia entre grupos con respecto a la media del tamaño del folículo dominante y a la media del tamaño del cuerpo lúteo al día 7 pos-cópula. Las variables tasa de ovulación y recuperación embrionaria fue evaluada con la prueba Chi-cuadrado y la calidad embrionaria entre los grupos de estudio fueron evaluadas con la prueba Kruskal-Wallis. El nivel de significancia empleado para el análisis de los resultados fue de $\mathrm{p}<0.05$.

\section{Resultados}

El tamaño del folículo dominante en el momento del servicio fue similar para los grupos tratados (Cuadro 1). Así mismo, 51 de las 53 alpacas servidas (96.2\%) presentaron ovulación al día 2 de la cópula, con formación de cuerpo lúteo al día 7 . La tasa de ovulación en $\mathrm{T}_{1}$ fue de $87.5 \%$ y de $100 \%$ en $\mathrm{T}_{2} \mathrm{y}$ $\mathrm{T}_{3}$, pero sin diferencia significativa entre tratamientos (Cuadro 2). Por último, el tamaño del cuerpo lúteo en el momento del lavado de cuernos uterinos fue similar para los tres grupos tratados (Cuadro 3).

Por otro lado, en el 52.94\% $(\mathrm{n}=27)$ de las alpacas servidas se recuperaron $27 \mathrm{em}-$ briones al día 7 de la monta, sin encontrar diferencias significativas entre tratamientos (Cuadro 4). No obstante, al comparar la calidad de los embriones según el estadio del folículo dominante en el momento de la cópula se observaron diferencias significativas entre $T_{1} y_{2} T_{2}$ con $T_{3}$, donde en este último se encontraron embriones de menor calidad $(\mathrm{p}<0.05)$ (Cuadro 5; Figura 2). 
Cuadro 1. Tamaño del folículo dominante en el día de la monta en alpacas

\begin{tabular}{lcccc}
\hline Tratamiento & $\begin{array}{c}\text { Hembras } \\
(\mathrm{n})\end{array}$ & $\begin{array}{c}\text { Promedio } \pm \\
\mathrm{DE}(\mathrm{mm})\end{array}$ & $\begin{array}{c}\text { Mínimo } \\
(\mathrm{mm})\end{array}$ & $\begin{array}{c}\text { Máximo } \\
(\mathrm{mm})\end{array}$ \\
\hline $\mathrm{T}_{1}$, Crecimiento & 16 & $7.81 \pm 0.31$ & 7.8 & 8 \\
$\mathrm{~T}_{2}$, Estática & 19 & $7.78 \pm 0.81$ & 7 & 10 \\
$\mathrm{~T}_{3}$, Regresión & 18 & $7.32 \pm 0.38$ & 7 & 18 \\
\hline
\end{tabular}

Cuadro 2. Tasa de ovulación en alpacas según el estadio de desarrollo de la onda folicular en el momento de la monta

\begin{tabular}{lccc}
\hline Tratamiento & $\begin{array}{c}\text { Hembras } \\
(\mathrm{n})\end{array}$ & $\begin{array}{c}\text { Tasa de ovulación } \\
(\%)\end{array}$ & $\begin{array}{c}\text { Hembras ovuladas } \\
(\mathrm{n})\end{array}$ \\
\hline $\mathrm{T}_{1}$, Crecimiento & 16 & 87.5 & 147 \\
$\mathrm{~T}_{2}$, Estática & 19 & 100 & 19 \\
$\mathrm{~T}_{3}$, Regresión & 18 & 100 & 18 \\
\hline Total & 53 & 96.2 & 51 \\
\hline
\end{tabular}

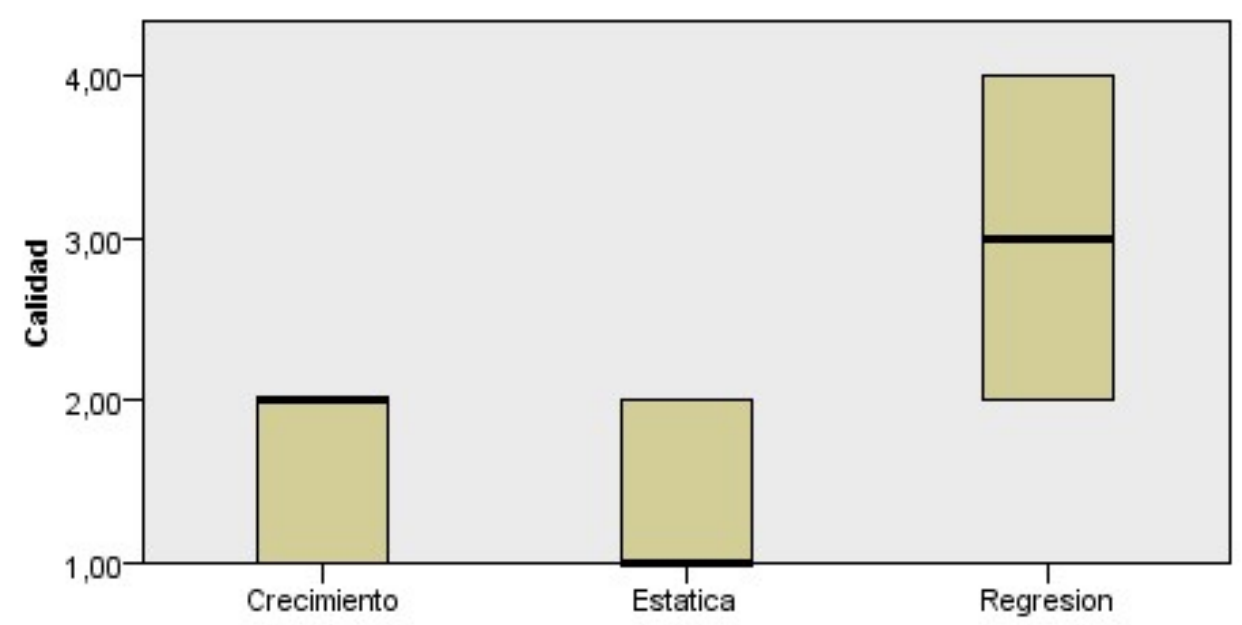

Figura 2. Relación entre calidad del embrión de alpaca y el estadio de desarrollo del folículo dominante en el momento de la monta (prueba de Kruskal-Wallis) 
Cuadro 3. Tamaño del cuerpo lúteo en el día 9 de la cópula en alpacas

\begin{tabular}{lcccc}
\hline Tratamiento & $\begin{array}{c}\text { Hembras } \\
(\mathrm{n})\end{array}$ & $\begin{array}{c}\text { Promedio } \pm \\
\mathrm{DE}(\mathrm{mm})\end{array}$ & $\begin{array}{c}\text { Mínimo } \\
(\mathrm{mm})\end{array}$ & $\begin{array}{c}\text { Máximo } \\
(\mathrm{mm})\end{array}$ \\
\hline $\mathrm{T}_{1}$, Crecimiento & 14 & $10.00 \pm 1.66$ & 7 & 13 \\
$\mathrm{~T}_{2}$, Estática & 19 & $10.05 \pm 1.31$ & 8 & 14 \\
$\mathrm{~T}_{3}$, Regresión & 18 & $10.09 \pm 2.01$ & 8 & 14 \\
\hline
\end{tabular}

Cuadro 4. Recuperación de embriones mediante el lavado de cuernos uterinos en alpacas

\begin{tabular}{lccc}
\hline Tratamiento & $\begin{array}{c}\text { Hembras } \\
(\mathrm{n})\end{array}$ & $\begin{array}{c}\text { Tasa de recuperación } \\
(\%)\end{array}$ & $\begin{array}{c}\text { Embriones } \\
\text { recuperados } \\
(\mathrm{n})\end{array}$ \\
\hline $\mathrm{T}_{1}$, Crecimiento & 14 & 64.3 & 9 \\
$\mathrm{~T}_{2}$, Estática & 19 & 47.4 & 9 \\
$\mathrm{~T}_{3}$, Regresión & 18 & 50.0 & 9 \\
\hline Total & 51 & 52.94 & 27 \\
\hline
\end{tabular}

Cuadro 5. Calidad de los embriones según el estadio del folículo dominante en el momento de la monta en alpacas

\begin{tabular}{lccccc}
\hline \multirow{2}{*}{ Calidad } & \multicolumn{3}{c}{ Estadio (tratamiento) } & \multicolumn{3}{c}{ Total } \\
\cline { 2 - 6 } & Crecimiento & Estática & Regresión & $\mathrm{n}$ & $\%$ \\
\hline Excelente & 4 & 6 & 0 & 10 & 37.0 \\
Buena & 5 & 3 & 3 & 11 & 40.7 \\
Regular & 0 & 0 & 3 & 3 & 11.1 \\
Mala & 0 & 0 & 3 & 3 & 11.1 \\
\hline Total & 9 & 9 & 9 & 27 & 100.0 \\
\hline
\end{tabular}

\section{Discusión}

Los resultados sugieren que las tasas de ovulación y de recuperación embrionaria no estarían afectadas por el estadio del desarrollo de la onda folicular al momento de la monta en alpacas. Si bien se encontraron algunas diferencias entre grupos (Cuadros 2, 3 y 4), estas no fueron significativas.
Bravo et al. (1991) indican que hembras con folículos mayores a $7 \mathrm{~mm}$ inducen la secreción de cantidades de LH hipofisiaria $(55.1-62.4 \mathrm{ng} / \mathrm{ml} / \mathrm{h})$ suficiente para producir la ovulación y formación de un cuerpo lúteo normal. La falta de ocurrencia de ovulación en los dos animales de $\mathrm{T}_{1}$ podría ser explicada si se considera que la duración total del estadio de crecimiento es de $4.8 \pm 1.5$ días 
(Bravo et al., 1990) y que la tasa de crecimiento es de $0.43 \pm 0.02 \mathrm{~mm} /$ día (Sumar et al., 1993), de allí que los folículos de estos animales aun no adquieren la sensibilidad debida a la LH para la ocurrencia de la ovulación, aunque también pudo haberse debido a un inadecuado estímulo de la monta, según lo señala Fernández-Baca et al. (1970).

La tasa de ovulación en la alpaca es alrededor del 90\% (Ratto et al., 2003), valor que concuerda con el $96.2 \%$ obtenido en el presente estudio. El tamaño promedio del cuerpo lúteo fue similar en los tres grupos, sin diferencia significativa al día 7 después de la cópula.

La diferencia en la calidad embrionaria por el estadio de desarrollo en que se encontraba el folículo dominante en el momento de la cópula podría ser explicada porque un folículo en regresión posiblemente contribuye a modificar el ambiente folicular, afectando la calidad del ovocito. Si bien no parece afectarse la capacidad de fecundación, pueden afectar la capacidad del desarrollo embrionario temprano pos-fecundación debido a la expresión de genes que contribuyen al desarrollo embrionario, lo cual ha sido reportado por Cordova et al. (2014) en estudios in vitro con ovocitos bovinos. Así mismo, Cervantes (2004) reporta que alpacas con presencia de folículos dominantes en estadio de regresión presentaban una menor tasa de sobrevivencia embrionaria.

\section{Conclusiones}

- La tasa de ovulación y el tamaño de cuerpo lúteo no se ve afectada por el estadio de desarrollo de la onda folicular en el momento de la cópula.

- La calidad de los embriones se ve afectada cuando la cópula se realiza en elmomento que el folículo dominante se encuentra en el estadio de regresión.

\section{Literatura Citada}

1. Bravo PW, Fowler ME, Stabenfeldt GH, Lasley BL. 1990. Ovarian follicular dynamics in the llama. Biol Reprod 43: 579-585. doi: 10.1095/biolreprod43.4.579

2. Bravo PW, Stabenfeldt GH, Lasley BL, Fowler ME. 1991. The effect of ovarian follicular size on pituitary and ovarian responses to copulation in domesticated South American camelids. Biol Reprod 45: 553-559.

3. Cervantes F. 2004. Estudio del efecto del estadio del desarrollo folicular al momento de la monta sobre la ovulación y sobrevivencia embrionaria en alpacas. Tesis de Médico Veterinario. Lima: Univ. Nacional Mayor de San Marcos. 53 p.

4. Cordova A, Perreau C, Uzbekova S, Ponsart C, Locatelli Y, Mermillod P. 2014. Development rate and gene expression of IVP bovine embryos cocultured with bovine oviduct epithelial cells at early or late stage of preimplantation development. Theriogenology 81: 1163-1173. doi: 10.1016/ j.theriogenology.2014.01.012

5. Fernández-Baca S. 1971. La alpaca. Reproducción y crianza. Bol 7. Lima: IVITA, UNMSM. 43 p.

6. Fernández-Baca S. 1991. Avances y perspectivas del conocimiento de los camélidos sudamericanos. Santiago de Chile: FAO. 429 p.

7. Fernández-Baca S. 1993. Manipulation of reproductive functions in male and female new world camelids. Anim Reprod Sci 33: 307-323. doi: 10.1016/ 0378-4320(93)90121-7

8. Fernández-Baca S, Maden DH, Novoa C. 1970. Effect of different mating stimuli on induction of ovulation in the alpaca. J Reprod Fertil 22: 261267. doi: 10.1530/jrf.0.0220261

9. Fernández-Baca S, Novoa C, Sumar $J$ 1972. Actividad reproductiva en la alpaca mantenida en separación del macho. Resúmenes de la Reunión de la Aso- 
ciación Latinoamericana de Producción Animal. Colombia: ALPA.

10. Huanca T. 2008. Efecto de la administración de gonadotropinas exógenas (FSH y eCG) en la respuesta ovárica y en la producción de embriones en alpacas (Vicugna pacos). Tesis Doctoral. Lugo, España: Univ. de Santiago de Compostela. $78 \mathrm{p}$.

11. Huanca W, Cordero A, Huanca T, Cardenas O, Adams GP, Ratto MH. 2009. Ovarian response and embryo production in llamas treated with equine chorionic gonadotropin alone or with a progestin-releasing vaginal sponge at the time of follicular wave emergence. Theriogenology 72: 803-808. doi: 10.1016/j.theriogenology.2009.05.019

12. Marín JC, Zapata B, González BA, Bonacic C, Wheeler JC, Casey C. 2007. Systematics, taxonomy and domestication of alpacas and llamas: new chromosomal and molecular evidence. Rev Chil Hist Nat 80: 121-140.

13. Novoa C. 1991. Fisiología de la reproducción de la hembra. En: Fernández-Baca $\mathrm{S}$ (ed). Avances y perspectivas del cono- cimiento de los camélidos sudamericanos. Santiago de Chile: FAO. p 93-103.

14. Ratto MH, Singh J, Huanca W, Adams GP. 2003. Ovarian follicular wave synchronization and pregnancy rate after fixed-time natural mating in llamas. Theriogenology 60: 1645-1656. doi: 10.1016/S0093-691X(03)00176-6

15. Ratto MH, Silva ME, Huanca W, Huanca T, Adams GP. 2013. Induction of superovulation in South American camelids. Anim Reprod Sci 136: 164-169. doi: 10.1016/j.anireprosci.2012.10.006

16. Stringfellow D, Givens D. 2011. Manual de la Sociedad Internacional de Transferencia de Embriones. $4^{\mathrm{a}}$ ed. Illinois, EEUU: International Embryo Transfer Society. $185 \mathrm{p}$.

17. Sumar J, Bravo PW, Foote WC. 1993. Sexual receptivity and time of ovulation in alpacas. Small Ruminant Res 11: 143-150. doi: 10.1016/0921-4488(93)90147-A

18. Sumar J. 2000. Llamas and alpacas. In: Hafez ESE (ed). Reproduction in farm animals. USA: Lippincott Williams \& Wilkins. p 218-228. 